\title{
The Translation Strategy of Advertisement Based on Nonequivalence between Chinese and English Conceptual Metaphors
}

\author{
Hongjun Chen ${ }^{1}$, Ruoyi Qiu ${ }^{1} \&$ Yu Wang ${ }^{2}$ \\ ${ }^{1}$ School of Foreign Languages, Dalian University of Technology, China \\ ${ }^{2}$ School of Software, Dalian University of Technology, China \\ Correspondence: Hongjun Chen, No. 2 Linggong Road, Ganjingzi District, Dalian, 116024, Liaoning Province, \\ China. E-mail: chenhj@dlut.edu.cn
}

\author{
Received: August 7, 2013 Accepted: September 9, 2013 Online Published: September 23, 2013 \\ doi:10.5539/ijel.v3n5p29 URL: http://dx.doi.org/10.5539/ijel.v3n5p29
}

\begin{abstract}
This article performed a qualitative and quantitative comparative study on metaphor translation between Chinese and English advertisements under the guidance of the Conceptual Metaphor Theory. Through an analysis of 100 pieces of well-known English advertisements and their official Chinese translation, the study found that the reflections of nonequivalence between Chinese and English metaphor embodied in the difference or missing of target domain in metaphoric mapping on one hand and the preference of conceptual metaphor types on the other. The purpose of reducing the information loses during the metaphorical conversion can be obtained by properly handling the mapping including adjustment, additions and omissions, and then adjusts the metaphor types according to the culture orientation and cognitive system of the target language. Such strategies can help better highlight the advertising purpose while maintain the poetic function of advertising at the same time.
\end{abstract}

Keywords: conceptual metaphor, metaphor translation, nonequivalence, Chinese and English advertisement

\section{Introduction}

Metaphor is pervasive in our everyday life and is a very important cognitive tool for us to understand the world. Conceptual metaphor theory is a milestone in metaphor research, first introduced by Lakoff and Johnson in 1980. According to the Conceptual Metaphor Theory, metaphor is fundamentally conceptual in nature and our everyday thought is largely metaphorical. Conceptual metaphors are systematic mapping across conceptual domains (Lakoff, 1993): the source domain of experience is mapped onto another domain of experience, the target domain. In this way, we understand or experience one thing in terms of the other.

The translation process of metaphor starts from the words and sentences of source language and ends with the words and sentences of the target language. But in the middle of this process is the complex cognition based on the translator's cultural embodiment. This is because that "embodiment and cognition comes before translation activity and the translated context is the fruit of embodiment and cognition. Translation is based on the translator's understanding of various meanings of the source context, so there is no doubt that understanding comes from embodiment and cognition" (Wang, 2005). Therefore, the nonequivalence becomes the biggest barrier to the translation of conceptual metaphor as due to the differences on social background, culture, traditions, language and even history and geography between two cultures. Under the guidance of the Conceptual metaphor theory, this paper does a systematical and comparative analysis between English advertisements and their translated version on mapping and metaphor types respectively.

\section{Literature Review}

The conversion of metaphor is one of the most important tasks in the translation field. The essay Can Metaphor be translated by Dagut in 1976, is regarded as an initial discuss of the translation of metaphor. A further essay the limits of translatability exemplified by metaphor translation by Van Den Broeck in 1981 conceives the treatment of metaphor as a functional relevancy to the communicative situation. Newmark (2001) first proposed the term "under-translation" in his book, referring to the original information being neglected or cut off in translated language causing readers unable to get necessary information for comprehension. Other commonly used strategies for metaphor translation are represented by Schaffner's (2004) generalization as substitution, paraphrase and deletion. Yet, the translation study in the west has always been advocating "the equivalent theory" 
among which Nida's theory of functional equivalence is the most familiar one to Chinese scholars. The translation with such a high degree of equivalence is very rare, unless the source text has little or no aesthetic value, such as tool manual or reference book.

Since Lakoff \& Johnson (1980) proposed the well-known embodied philosophy based on the reflection and criticism of the traditional empiricism and rationalism, cognitive linguistics are bringing an increasingly important influence to the studies of translation. Based on this cognitive perspective of language and the conceptual metaphor theory, it is the same objective world of all nations as well as similar physiological basis and cognitive ability of cultural exchanges among different nations that lead to the similarity and translatability of cognitive systems. However, there is also difference in cognitive embodiment of various nations derived from different cultures and ideologies, it is exactly such difference of embodiment hindering the conversion of metaphor (Xiao \& Li, 2010). There are both equivalence and nonequivalence in Chinese and English conceptual metaphor systems. In translation process, to be exactly, the nonequivalence of metaphor come from the difference of cultural background, tradition, ideology and other inner factors between writer and translator. Since the translation of metaphor is a cognitive process based on translator's cultural embodiment, the basis of metaphor translation is the overlap of cultural experience in different nations. Chinese and Western cultures have their respective origins while they also exchange and integrate in the long history. As a result the conceptual metaphor systems of Chinese and English have both equivalency and nonequivalence.

The nonequivalence between Chinese and English conceptual metaphors are well-noticed by Chinese scholars and as a result theories like functional equivalence theory and co-relation theory prevailed in the west are questioned and challenged especially in translating special texts like, advertisement. The study of metaphorical advertisement translation is brand new compared with studies of metaphor in literature. The translation of advertisement naturally was consumer-oriented. Through advertisement, consumers get to know the characteristics, performance and use of the products or even the corporate cultures of producers. Metaphors, functioned as a means of cognition, play an important role in persuading consumers to buy their products. Thus, a successful advertisement translation must have the ability to recreate the association of the original image within the culturally cognitive system of the target consumers. Obviously the equivalence theory is not suitable for the translation of advertisement, especially metaphorical advertisement.

But, current researches are restricted on the rhetorical level and the theoretical basis is limited to the traditional translation theories. Most data used are random rather than based on corpus statistical analysis. Particularly, the decisive factor of conceptual metaphor translation is not revealed in translation strategies. This article performed a qualitative and quantitative comparative study on metaphor translation through analyzing 100 pieces of well-known English advertisements and their official Chinese translation to locate the translation strategies of nonequivalence between Chinese and English advertisements.

\section{Methodology}

The English advertisement are collected from http://www.textart.ru/database/slogan/list-advertising-slogans.html. Most of the Chinese advertisements are collected from http://www.zhengjicn.com/zy/ggy/index.html. Among thousands of advertisements both Chinese and English, 100 were chosen out for official translated version. After being crosschecked on their official websites, these 100 pieces of advertisement and their official translated version make up the corpus of this thesis. Discussion, including case studies for revealing the causes and reflection of the nonequivalence between Chinese and English conceptual metaphors and the following qualitative and quantitative analysis are all based on this corpus.

The 100 pieces of advertisement and their translated version are analyzed in two steps, i.e., analysis of mapping and conceptual metaphor types. To know a conceptual metaphor is to know the set of mapping that applies to a given source-target pairing. The analysis of mapping is the foundation of the analysis of conceptual metaphors. According to cognitive linguistics, there are two main roles for the conceptual domains posited in conceptual metaphors: Source domain; the conceptual domain from which we draw metaphorical expressions and Target domain: the conceptual domain that we try to understand. A mapping is the systematic set of correspondences that exist between consistent elements of the source and the target domain.

\section{Results and Discussion}

Lakoff and Johnson differentiate three types of metaphors: structural, orientational, and ontological metaphor. With the specific examples of translation between Chinese and English metaphorical advertisement, we summarize some strategies for dealing with the nonequivalence of metaphor types in metaphorical advertisement respectively from the three categories of conceptual metaphor types. 


\subsection{The Translation Strategies for Dealing with the Nonequivalence of Structural Metaphor}

Structure metaphor is the basis for the other types of conceptual metaphor. Advertisement, especially metaphorical advertisement can help consumers to obtain anticipation and an overall understanding of the products' quality, function and feeling of use before purchasing. However, the reciprocity in the translation of metaphorical advertisement is very difficult to achieve due to the nonequivalence between Chinese and English structural metaphors. Even under the precondition that some metaphor concepts are very similar in Chinese and English cognitive systems, the nonequivalence in the preference of metaphor types still can cause problems such as the unacceptable translation or improper association of the image to readers in the target language. Analyzing 100 pieces of advertisements of world-known brands and their official Chinese version, we summarize the translation results of structural metaphors in the original advertisements as in the following chart:

Table 1. The translation of structural metaphor

\begin{tabular}{|l|l|lc|}
\hline & & Translated Ads with Structural Metaphor & 32 \\
\cline { 3 - 3 } $\begin{array}{l}\text { The amount of } \\
\text { original Ads with } \\
\text { structural Metaphor }\end{array}$ & 42 & Translated Ads with Ontological Metaphor & 2 \\
\cline { 3 - 3 } & & Translated Ads with Orientational Metaphor & 0 \\
\cline { 2 - 3 } & Translated Ads with no Conceptual metaphor & 8 \\
\hline
\end{tabular}

As listed in Table 1, there are 32 pieces of translated advertisements maintaining the structural metaphor type in the translation process. We found that four methods are often used in the translation of structural metaphor between English and Chinese metaphorical advertisements. (1) The first method is to keep the structural metaphor if the metaphor concept is universal. Take "the heat beat of America (Chevrolet)" as example. The original advertisement contains the structural metaphor with a mapping from engine to heart beat. The translation of this piece of advertisement is “雪佛兰, 美国的动力之源”. It retains the structural metaphor type but changes the original mapping to a new mapping that is more familiar with Chinese consumers that is mapping from "engine" to "source of power". Furthermore, we found that structural metaphor has a very obvious feature that it is always conveyed by the word "之" in Chinese. As such, the translators use this form of expression as “动力之源”. (2) The second method is to keep the metaphor type and mapping of structural metaphor the same and use “像”, “如” and words like that to change the original structural metaphor into simile. In such way, the rhetoric use of simile can better interpret the metaphor concept in the original metaphorical advertisement. There are many example of this methods like "Blessed by year round good weather, Spain is a magnet for sun worshippers and holidaymakers” (西班牙蒙上帝保佑, 一年四季, 天气很好, 宛如一块磁铁, 吸引着酷爱阳光、喜爱度假的人们). This is a piece of tourism advertisement. The original advertisement contains the metaphor concept "Spain is magnet". The translated version reproduced the metaphor concept by simile with the start of “宛如”. Another example is “Waterford, Light a Crystal Fire” (瓦特福水晶玻璃器血, 像 一团透明的火焰). Here the original piece of advertisement also has structural metaphors with both mapping to crystal and mapping to fire. While the in the translation process, the translator use a simile “像一团透明的火焰” instead of the structural metaphor. Although the mapping to crystal is omitted, the mapping to fire is highlighted and at the same time the crystal and sparkling characteristics of the product are not by a fraction shaded. (3) The third method is called "literal translation". Literal translation is thought by some scholars to be the most essential and the most commonly used method for translating metaphors. However, as we have mentioned in the previous discussion, to fit the cultural embodiment of the target consumers and affected by the translator's cognitive level, literature level and aesthetic level, the literal translation of metaphorical advertisement is really rare. Only when the original advertisement conveys one mapping universally comprehended by readers from target language without any difficulty, confusion or ambiguity can the translator literally translate the advertisement. Some examples are like “Bread is life" (面包就是生活) for BICE bread and “Source of enchantment” (诱惑之源) for Lancome perfume. Since this approach is limited to the overlapping part of conceptual metaphors in Chinese and English cognitive systems, the application of this method is rare and requires careful interpretation. (4) Last feature in the translation between Chinese and English metaphorical advertisements is to omit the structural metaphor so as to help the target consumers get a more direct and accurate perception of the product. Take "the most unforgettable women in the world wear Revlon" as example. This piece of advertisement contains a structural metaphor that has a mapping from "cosmetics" to "clothes". Since this brand of cosmetics is very popular in America, the consumers in America are clear that the product in the advertisement is cosmetics, not 
clothes. However the consumers of the target language are not that familiar with the product, which will lead Chinese consumers to the confusion that what the product is. To avoid confusion and vagueness, the translator omitted the metaphor concept and translated advertisement into “世界上最令人难忘的女人都用露华浓”. Although the structural metaphor is omitted, the essence and functions of the advertisement are achieved in this way.

Structural metaphors are also changed into ontological metaphors for a better translation. Under such circumstances, the metaphor concept either keeps unchanged or deleted completely, such as the advertisement for ISDN communication company, "Free your enterprise from the communication prison". Its Chinese translation version is “让你的公司免做通讯的风犯”. The metaphor concept “communication barrier is a prison” keeps unchanged in the translated version. Yet, the Chinese version changes the mapping from the company to prisoner, this personification group the translation into the category of ontological metaphor. Another example is the advertisement for LANCOME, "Skin looks as if lit from within - breathtakingly radiant. Skin is cushiony soft and velvety to the touch (看得见的弹润透亮，摸得到的柔软细淢)”. The metaphor concept is deleted completely in this translation process. The conceptual metaphors "skin is cushion" and "skin is velvet" in the original advertisement are thoroughly omitted and replaced by the materialization of "soft" and "delicate" of the entity which fell into the category of ontological metaphor.

However, there are rare cases where the structural metaphor is changed into orientational metaphor in the translation between Chinese and English metaphorical advertisement due to the limitations and commonality of physical experiences and spatial experiences.

\subsection{The Translation Strategies for Dealing with the Nonequivalence of Ontological Metaphor}

The ontological metaphor can help people to see abstract and vague thoughts, feelings, mental activity and other intangible concepts as concrete and tangible entities. Ontological metaphors specify different kind of objects. They give us different metaphorical models for what the mind is and thereby allow us to focus on different aspects of mental experience. Since advertisement also has such cognitive function as to specify the nature, functions and characteristics of the product, ontological metaphor is the most commonly used metaphor type in advertisement.

Table 2. The translation of ontological metaphor

\begin{tabular}{|l|l|lc|}
\hline & & Translated Ads with Structural Metaphor & 8 \\
\cline { 2 - 3 } $\begin{array}{l}\text { The amount of } \\
\text { original Ads with }\end{array}$ & 44 & Translated Ads with Ontological Metaphor & 23 \\
$\begin{array}{l}\text { Ontological } \\
\text { Metaphor }\end{array}$ & & Translated Ads with Orientational Metaphor & 0 \\
\cline { 3 - 4 } & & Translated Ads with no Conceptual metaphor & 13 \\
\hline
\end{tabular}

Through the statistic analysis, we can summarize the translation results of ontological metaphors in the original advertisements as in Table 2.

The translation of ontological metaphor Lakoff and Johnson (1980) further divided the ontological metaphor into three subtypes in their masterpiece "Metaphors we live by" as container metaphor, entity and substance metaphor, as well as personification. In the book, Lakoff and Johnson states that: Each of us is a container, with a bounding surface and an in-out orientation. We also view them as containers with an inside and an outside. Lakoff and Johnson (1980) also state that perhaps the most obvious ontological metaphors are those where the physical object is further specified as being a person. This allows us to comprehend a wide variety of experiences with nonhuman entities in terms of human motivations, characteristics, and activities. We call these metaphors personification. For entity and substance metaphor, they point out in the book that understanding our experiences in terms of objects and substances allows us to pick out parts of our experience and treat them as discrete entities or substances of a uniform kind. Once we can identify our experiences as entities or substances, we can refer to them, categorize them, group them, and quantify them and, by this means, reason about them. 
Table 3. The translation of the subtypes of ontological metaphor

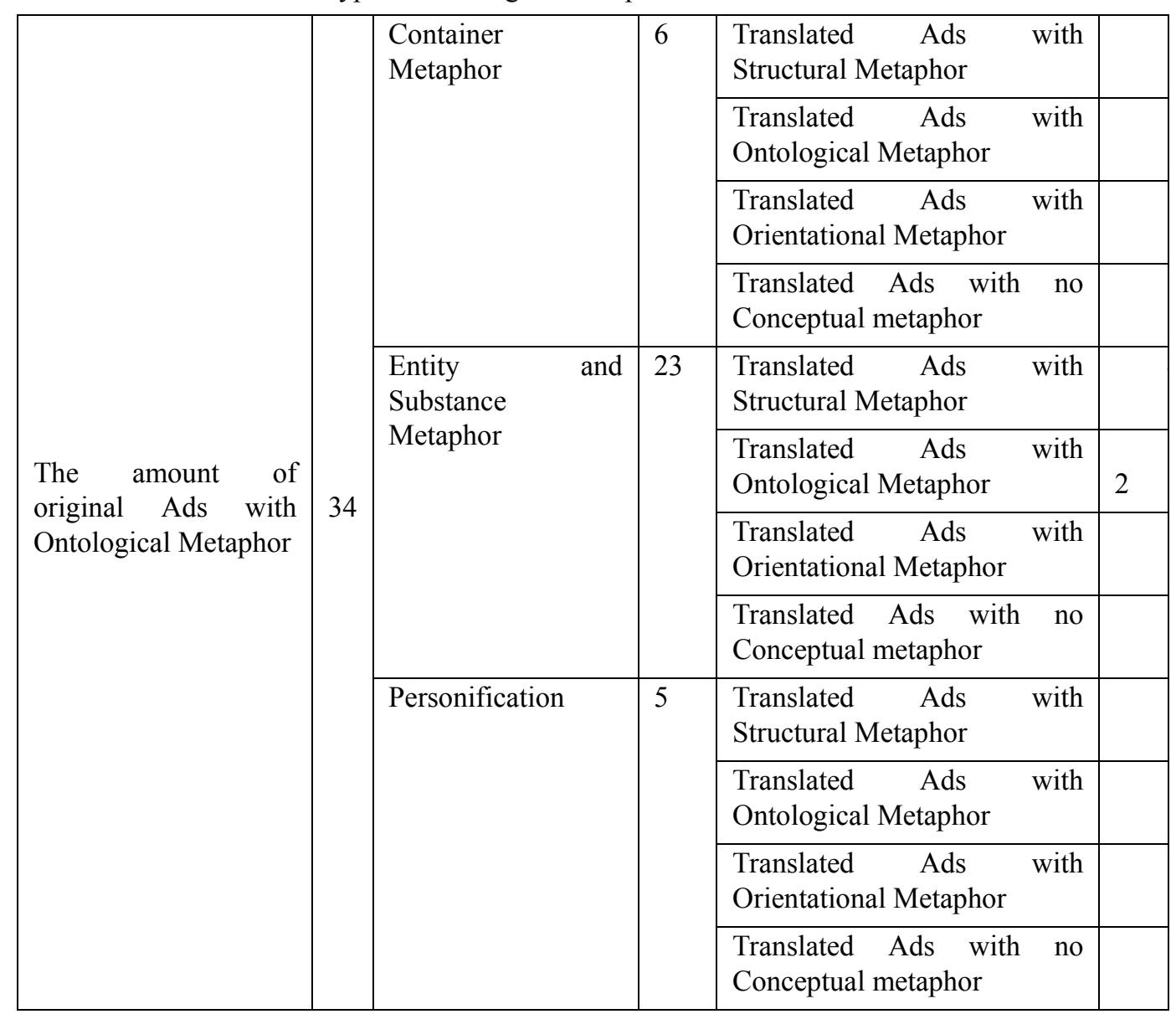

Analyzing the 100 pairs of chosen advertisements, we can reveal the translation results of the subtypes of ontological metaphor. As can be seen in Table 3, the number of entity and substance metaphor is the highest and the number of entity and substance metaphor and personification is much higher than container metaphor.

Firstly, we found that in the translation process, all the three subtype of ontological metaphor have examples of being converted into structural metaphor.

For example the advertisement “Engineered to move the human spirit (人类精神的动力)" for Mercedes - Benz is the typical conversion from entity and substance metaphor to structural metaphor. The original metaphorical advertisement has a entity and substance metaphor which use the verb "move" to materialize the "human spirit". While the translated version omit the verb "move" and instead use a structural metaphor "car is human spirit" to better highlight the mapping and achieve the function of the advertisement that reveal the pioneering and innovative corporate culture of Mercedes-Benz.

Let us see another example of conversion from personification to structural metaphor in the translation of metaphorical advertisement between Chinese and English: "Feast your eyes (滋润心灵的窗户)” for Pond's. With the word "feast", the original text obviously has a personification belonging to ontological metaphor, mapping from the "eyes" to the "treatable guest". There is nonequivalence of culture embodiment since such mapping is unfamiliar with Chinese consumers, so the translator decisively choose to rewrite the original mapping into "the eyes are the windows" which is more familiar and acceptable with Chinese consumers. As a result the metaphor type also changes from personification to the structural metaphor. At the same time the translation maintains the poetic effect in the original advertisement and use the word "滋润 (moisture)" to point out the function of the product. This is a very successful example of handling metaphor types in the translation of metaphorical advertisement.

A typical example for the conversion from the container metaphor to structural metaphor is the advertisement “In Search of Excellence (别克轿车, 志在千里)" for Buick. In the original advertisement, the activity "search" is comprehended through ontological metaphor with the state "search of excellence" as a container. However, the 
translated version changes this container metaphor into structural metaphor in order to better highlight the essence of advertisement. "Excellence" is the essence of original advertisement while to Chinese consumers it is a very abstract and vague concept. Instead there is an allusion about a winged steed in the ancient Chinese culture and the association between the ancient means of transport "horse" and the modern means of transport "car" understanding is not far-fetched at all. Besides, this structural metaphor gives the advertisement a more cultural and historical depth. The common ground for the translator to change the ontological metaphor into structural metaphor in the translation of metaphorical advertisement is that ontological metaphor is not used for conveying the essence of the advertisement and such ontological metaphor can be processed into a more clear structure metaphor or deleted completely in the translation of metaphor. Such as this following piece of advertisement - "Green shadow which accompanies you and its translated Chinese version (随身的绿荫)". The English advertisement has both the structural metaphor with the mapping from "shadow" to "cosmetics" and the ontological metaphor which further fell into the category of personification carried by the word "accompany". While in the translation process, the ontological metaphor is omitted and the structural metaphor is retained to highlight the essence of the advertisement. Although there can be two kinds of understanding of the word "accompany" as one is "caring along with" and the other is "following you around". The ontological metaphor in the English advertisement obviously applies the second interpretation of "accompany", but consumers' attention will be diverted if the ontological metaphor literally translated since the difference of word order between Chinese and English will make the ontological metaphor be ahead of structural metaphor in the translated advertisement. So in order to highlight the more important mapping that is from "shadow" to "cosmetics" which can help the consumer to focus on the function of the product is faithfully recreated as structural metaphor in the translation process.

As can be seen from the above analysis that the function of verb cannot be ignored in the process of ontological metaphor, and therefore verbs and verb phrases become the starting point in the translation strategies of ontological metaphors. One way to properly handle the nonequivalence of ontological metaphor in the translation process is to nominalize the verbs or verb phrases. Take the following advertisement of a magazine for example: "The world smiles with Reader's Digest". This piece of English advertisement contains the ontological metaphor, with the verb "smile" making the word "world" personified. However, the equivalent word in Chinese to "world" is “世界”. “世界” first appeared as a Buddhist terminology with “世” referring to the time and “界” referring to the space. So in traditional Chinese cultural embodiment there is seldom case to see the world as a person. To avoid such nonequivalence in the translation process, the translator converted the personification into entity and substance metaphor through the nominalization of the word "smile". As a result the Chinese translation “读者文摘给世界带来欢笑” contains an entity and substance metaphor that "laugh is something that can be brought about". The other way to properly handle the nonequivalence of ontological metaphor is the omission or obfuscation of verbs and verb phrases. For example the metaphorical advertisement for Rossini Watch - "Time always follows me". This piece of advertisement contains an ontological metaphor, with the verb “follow" personified the concept of “time”. However in the translated version “罗尼西表: 时间因 我而存在”, the translator omitted the verb “follow" and as a consequence the ontological metaphor is omitted because the nonequivalence of the metaphorical concept of "time" in Chinese and English cognitive systems. Although metaphorical concepts like "time is something that has value" and "time is water" are universal, Chinese culture seldom has expressions that containing "time follows people". It would be so much better to highlight the essence of advertisement than constrained to the formality. “因我而存在” better conveys individualism emphasized in the original advertisement.

Although ontological metaphors are widely applied in the advertisement, in the translation process of such metaphorical advertisement they are often converted into structural metaphors with clearer and more important mappings or omitted in order to highlight the essence of the advertisement. Ontological metaphor, especially personification, has a very frequent application in the English advertisement because of its significant contribution in increasing the interest, vivid and readable of advertisement. However in the translation process from English to Chinese, personification always is converted into entity and substance metaphor, structural metaphor or deleted completely in order to reduce the association failure. Same with the situations of conversion between structural metaphor and orientational metaphor, there are rarely any cases when the ontological metaphor is changed into orientational metaphor in the translation between Chinese and English metaphorical advertisement due to the limitations and commonality of physical experiences and spatial experiences.

\subsection{The Translation Strategies for Dealing with the Nonequivalence of Orientational Metaphor}

Lakoff and Johnson (1980) state in Metaphors we live by that there is another kind of metaphorical concept: one that does not structure one concept in terms of another but instead organizes a whole system of concepts with 
respect to one another. We will call these orientational metaphors. Orientational metaphor refers to the a series of metaphorical concepts formed on the reference of spatial orientation: up-down, in-out, front-back, on-off, deep-shallow, central-peripheral and has a tight connection with physical experience. These metaphor concepts appear in everyday life from time to time, but are not as widely used as the other types of metaphor in advertisements.

Table 4. The translation of orientational metaphor

\begin{tabular}{|l|l|l|l|}
\hline & Translated Ads with Structural Metaphor & 3 \\
\cline { 3 - 3 } $\begin{array}{l}\text { The amount of } \\
\text { original Ads with } \\
\begin{array}{l}\text { Orientational } \\
\text { Metaphor }\end{array}\end{array}$ & 9 & Translated Ads with Ontological Metaphor & 0 \\
\cline { 2 - 3 } & & Translated Ads with Orientational Metaphor & 5 \\
\cline { 2 - 3 } & Translated Ads with no Conceptual metaphor & 1 \\
\hline
\end{tabular}

Through the statistic analysis of one hundred pieces of advertisements of world-known brands and their official translated Chinese version, we summarize the translation strategy of orientational metaphors in the original advertisements as in Table 4.

Because different countries and different nationalities have basically the same physical experience and spatial orientations except for a few cases, such as in India, nodding stands for negative instead of positive as usual, while shaking one's head means yes. But with the development of globalization and internationalization, many physical experiences and spatial orientations are gradually being assimilated. China, Europe and the United States have basically the same spatial orientation and physical experiences. Therefore, if the metaphor type keeps unchanged, the nonequivalence of orientational metaphor in Chinese and English advertisement is not that much.

Through comprehensive analysis there are only two points to note in the translation process: 1) If the original metaphorical advertisement has both an orientational metaphor and an ontological metaphor, ontological metaphor is often omitted to highlight the orientation metaphor in the translation process. The example below can better illustrate this point: “It gives my hair a top-quality look (一流秀发, 超级精彩)”. The original advertisement contains both an orientational metaphor with the metaphorical concept "up is good" and an ontological metaphor which take "a look" as entity that can be "gives". 2) To deal with the nonequivalence between Chinese and English cognitive systems, sometimes we can transform the orientational metaphor into structural metaphor in the translation process, and more often than not combine the translation with the compensation strategy. For example "Start ahead (成功之路, 从头开始)" for Rejoice. The original English advertisement choose a crafty application of the word "ahead", which not only contains an orientational metaphor with the metaphorical concept as "front is good" but also points out the effectiveness and features of the product. However, due to the nonequivalence of language between Chinese and English, we cannot find an equivalent word with "ahead" in Chinese. So the translator applies the compensation strategy, creating a structural metaphor as “成功之路 (success is path)” in the translation process to interpret the deep meaning conveyed in the original advertisement. Moreover, the translator compensates the expression “从头开始” as a replacement of the pun usage of the word "ahead".

Due to the limitations on spatial orientations and body experiences, the application of orientational metaphors in the advertisement is much less compared with the other two metaphor types with the appearance in only 9 pieces out of 100 pieces of advertisements. Also as a result, there is little cases in which structural metaphors or ontological metaphors are converted into orientational metaphor when dealing with the nonequivalence of metaphor types between Chinese and English conceptual metaphors. But since orientational metaphor has the same characteristics with advertisement on directness, simplicity and other features of expression, it should not be ignored in the analysis and translation of metaphorical advertisement.

\section{Conclusion and Significance}

Through analysis of the reflections of nonequivalence between Chinese and English conceptual metaphors based on the comparison of English advertisement and its translated version, this paper found out that the reflection of the nonequivalence between Chinese and English conceptual metaphors can be divided into two kinds. One reflection is the nonequivalence of mapping, to be exactly is the difference or missing of target domain. The other is reflected on the preference in choosing metaphor types when the metaphor concept remains the same. 
Through the analysis of successful translation cases of some famous advertisements, this paper summarized that there are three strategies that can be applied in the translation process to properly handle the nonequivalence of mapping between Chinese and English advertisements, namely the adjustment of mapping, the increase of mapping and the ellipsis of mapping. In some metaphorical advertisements when Chinese and English metaphors have very little nonequivalence, we adjust the mapping by narrow down the source domain or extend the source domain. When the original metaphorical advertisement is too concise and may cause ambiguity or confusion of the metaphor, we increase mappings to better convey the purpose and function of the advertisement. When the conceptual metaphor is not used to convey the purpose and function of the advertisement which more likely to has more than one metaphor concepts or we can say when the metaphor is more like an assistant than essence, we delete the mapping and use fuzzy process of four-character or N-character words and idioms. Then after the interpretation and recreation of mapping, we need to find some ways for proper handling of the nonequivalence on metaphor types between Chinese and English conceptual metaphors. Through quantitative and qualitative analysis of metaphorical advertisements, this paper summarized some strategies to deal with the nonequivalence between Chinese and English conceptual metaphor types in order to reproduce the purpose and essence of the original advertisement while maintaining the poetic function with the help of language characteristics of Chinese and English. This paper summarized that if the original advertisement contains the structural metaphor the translation preferred to maintain the type with three methods commonly used: (1) structural metaphor to structural metaphor with the application of the strategies to handle the nonequivalence of mapping; (2) keep the metaphor type and mapping of structural metaphor the same and use “像”, “如” and words like that to change the original structural metaphor into simile; (3) literal translation with the purpose to reduce the information loses as much as possible. For the other cases, the translation between Chinese and English metaphorical advertisements sometimes omit the structural metaphor based on the principle of obfuscation in order to help the target consumers to grasp the essence of the advertisement and get a more direct and accurate perception of the product. In the ellipse of structural metaphor, Chinese translated advertisements also commonly use four-character or N-character words as ways of fuzzy process. Moreover, the structural metaphors in the original text also can be changed into ontological metaphors and under such circumstances, the metaphor concept either keeps unchanged or deleted completely. Although ontological metaphors are widely applied in the advertisement, in the translation process of such metaphorical advertisement they are often converted into structural metaphors with clearer and more important mappings or omitted in order to highlight the essence of the advertisement. Ontological metaphor, especially personification, has a very frequent application in the English advertisement because of its significant contribution in increasing the interest, vivid and readable of advertisement. However in the translation process from English to Chinese, personification always is converted into entity and substance metaphor, structural metaphor or deleted completely in order to reduce the association failure. For orientational metaphor, this paper summarize two points 1) If the original metaphorical advertisement has both an orientational metaphor and an ontological metaphor, ontological metaphor is often omitted to highlight the orientation metaphor in the translation process. 2) To deal with the nonequivalence between Chinese and English cognitive systems, sometimes we can transform the orientational metaphor into structural metaphor in the translation process, and more often than not combine the translation with the compensation strategy. Due to the limitations on spatial orientations and body experiences, the application of orientational metaphors in the advertisement is much less compared with the other two metaphor types, also because of this reason there are rarely any cases when the structural metaphor and ontological metaphor is changed into orientational metaphor in the translation between Chinese and English metaphorical advertisement.

This study is helpful for the cross-cultural communication as well as language teaching and learning. By comparative study of the equivalence and nonequivalence between Chinese and English cognitive systems and language systems, the learners can get a better understanding of both the characteristics of English and Chinese languages and inherent connection between culture and metaphor. Also it can test the universality of the conceptual metaphor theory and enrich the corpus for the analysis of conceptual metaphor.

\section{Acknowledgement}

This study is part of a project on metaphor funded by Humanities and Social Sciences Research Project under the Ministry of education of China (12YJCZH010).

\section{Reference}

Lakoff, G. (1993). The Contemporary Theory of Metaphor. Metaphor and Thought. Cambridge: Cambridge University Press.

Lakoff, G., \& Johnson, M. (1980). Metaphors We Live by. Chicago: The University of Chicago Press. 
Newmark, P. (2001). Approaches to Translation. Shanghai: Shanghai Foreign Language Education Press.

Schäffner, C. (2004). Metaphor and translation: Some implications of a cognitive approach. Journal of Pragmatics, 2004(36), 1254-1269.

Wang, Y. (2005). Translation models from cognitive linguistics perspective. China Translation, 2005(5), 15-20.

Xiao, J. Y., \& Li, H. W. (2010). Metaphor translation Research from the conceptual metaphor perspective. Foreign Languages in China, 7(5), 106-111.

\section{Copyrights}

Copyright for this article is retained by the author(s), with first publication rights granted to the journal.

This is an open-access article distributed under the terms and conditions of the Creative Commons Attribution license (http://creativecommons.org/licenses/by/3.0/). 